Objective The main objective was to determine incidence of sepsis and outcome in neonates treated in the PICU, Department of Paediatrics, University Hospital of Split, Croatia.

Methods All neonates hospitalized in the period from 1st January 2003 to 31st December 2018 were included in the research. Medical documentation was examined and data were analysed using MedCalc (MedCalc Software Inc., Ostend, Belgium). The statistical methods used for the quantitative data were median, interquartile ranges and Mann-Whitney test. Qualitative data were analysed with $\chi 2$ test. The results were interpreted at a significance level of $\mathrm{p}<0.05$.

Results Out of 771 treated neonates, 128 (16.6\%) had sepsis. Early-onset sepsis was diagnosed in 59 (46\%) and late-onset in $69(54 \%)$ neonates. Median birth weight was $2255 \mathrm{~g}$ (range 630-5000 g), while $85(66 \%)$ were male. There were $78.8 \%$ preterm neonates and $21.2 \%$ term neonates. The most common perinatal diagnoses were premature rupture of membranes (14.1\%) and chorioamnionitis (10.9\%). The most common reasons for hospital admission were intracranial hemorrhage (43.1\%) and RDS (39.1\%). Median of CRP value was $80.8 \mathrm{mg} / \mathrm{L}$ (range $1.2-382 \mathrm{mg} / \mathrm{L}$ ). The values of procalcitonin were high in $88.5 \%$ patients, $32 \%$ of subjects had leucopenia and $42,6 \%$ of them had leucocytosis. Trombocytopenia was found in $63 \%$ of patients. Gram-negative bacteria were isolated in $31.2 \%$ of subjects and gram-positive bacteria in $28.2 \%$ of subjects. Fungi were isolated in $6.5 \%$ of subjects, and $5.6 \%$ had more than one bacteria isolated (out of 3 hemocultures). The lumbar puncture was positive for meningitis in 12 patients (9.38\%). The majority of patients (78\%) were on invasive ventilation (SIMV, HFOV) as a part of treatment and median of length of invasive ventilation was 9 days (range 1-140). Median length of hospitalisation was 19 days (range 9-108). Out of a total number of patients, $23 \%$ died. The mortality rate in early-onset sepsis group was $27 \%$ and in late-onset sepsis group $19 \%$.

Conclusion The relatively high mortality in neonates with sepsis could be explained by the fact that the most seriously ill newborns from the general hospitals of the Dalmatia region are treated in our PICU. The values of CRP, length of invasive ventilation and length of hospitalisation had a statistically significant effect regarding the type of sepsis (early-onset or late-onset sepsis). It is important to recognize and treat neonatal sepsis in a timely manner in order to reduce the mortality rate as well as potential long-term consequences.

\section{EARLY NEONATAL HEALTH CARE OUTCOMES IN UNIVERSITY HOSPITALS AND UNIVERSITY HOSPITAL CENTERS VS. OTHER HEALTH INSTITUTIONS IN CROATIA}

Boris Filipović-Grčić* Željka Draušnik, Urelija Rodin, Ivan Cerovečki, Ruža Grizelj, Dorotea Ninković, Nada Sindičić Dessardo, Darjan Kardum. University Hospital Center Zagreb, University of Zagreb School of Medicine

\subsection{6/archdischild-2021-europaediatrics.118}

To compare early neonatal health care outcomes in university hospitals (including university hospital centers) with outcomes in other health institutions in Croatia.

An observational retrospective study of childbirth data from National Medical Birth Database was conducted. Outcomes (early neonatal mortality or survival rate) of all neonates of gestational age $<32$ completed weeks in the period 20092018 were studied with regard to birth settings (university hospitals and university hospital centers vs. other health institutions).

The statistical significance of differences in average early neonatal mortality rates (ENM) was determined.

In the period 2009-2018 there were 1,282 live-born children of gestational age 22-27 completed weeks in Croatia. Most (84.8\%) were born in university hospitals (rate increasing over the studied period) and the rest (15.2\%) in other health institutions. The respective average birth rates were 5.0/ 1,000 live births in university hospitals and 1.1/1,000 live births in other hospitals, with minor oscillations throughout the studied period (university hospitals: 4.0-6.3; other hospitals: $0.5-1.5)$. The average ENM rate in this gestational group was statistically significantly higher among neonates born in other hospitals $(565.7 / 1,000$ live births) compared to those born in university hospitals (373.7/1,000 live births), $\chi 2=25,316, \mathrm{P}<0.0001$. Out of

2,148 live-born children of the gestational age 28-31 completed weeks most were born $(87.8 \%)$ in university hospitals and the rest $(12.3 \%)$ in other hospitals. The respective average birth rates were $8.6 / 1,000$ in university hospitals (with an intermittently increasing rate) and $1.5 / 1,000$ live births in other hospitals. The average ENM rate in this gestational group was higher in other hospitals (91.2/1,000 live births) compared to university hospitals $(62.0 / 1,000$ live births), but with no statistically significant difference; $\chi 2=3.220$, $\mathrm{P}=0.0727$.

The ENM rate in children of gestational age 22-27 completed weeks is higher in other hospitals than in university hospitals. Furthermore, the proportion of these children born in university hospitals in Croatia has increased over the studied 10-year period. As pre-term birth (before 32 weeks of gestation in particular) is closely related to perinatal outcome (i.e. high mortality or long-term impairment), analysis of their outcomes is important for planning and development of an appropriate neonatal intensive care unit network as well as to improve decision-making processes in health politics.

\section{POSTOPERATIVE COMPLICATIONS AND THEIR PREDICTORS IN NICU PATIENTS WITH SURGICAL DIAGNOSIS}

Mirna Sipl*, Dalibor Divković, Ivana Sarić. University Hospital Centre Osijek, Clinic of Pediatrics, Neonatology Department

\subsection{6/archdischild-2021-europaediatrics. 119}

To investigate the incidence, type, severity and time of occurence of various postoperative complications in NICU patients undergoing surgery under general anesthesia and to determinate the preoperative, intraoperative and postoperative risk factors which can predicte their appearance.

We retrospectively analyzed the postoperative complications which occurred in neonatal/preterm patients with congenital or acquired surgical condition and whose surgery under general anesthesia was performed during hospitalisation in tertiary level NICU centre Osijek during five years period from January 2015 to December 2019.

Patients with congenital diaphragmatic hernia, esophageal atresia and congenital hearth disease were excluded because they were for treatment transported in higher, level IV NICU Centre, Zagreb. 